\title{
Matrix Games, Mixed Strategies, and Statistical Mechanics
}

\author{
J. Berg * and A. Engel ${ }^{\dagger}$ \\ Institute for Theoretical Physics \\ Otto-von-Guericke University, Postfach 4120, D-39016 Magdeburg, Germany
}

\begin{abstract}
Matrix games constitute a fundamental problem of game theory and describe a situation of two players with completely conflicting interests. We show how methods from statistical mechanics can be used to investigate the statistical properties of optimal mixed strategies of large matrix games with random payoff matrices and derive analytical expressions for the value of the game and the distribution of strategy strengths. In particular the fraction of pure strategies not contributing to the optimal mixed strategy of a player is calculated. Both independently distributed as well as correlated elements of the payoff matrix are considered and the results compared with numerical simulations.

PACS: 05.20-y, 02.50.Le, 64.60.C
\end{abstract}

Game theory models in mathematical terms problems of strategic decision-making typically arising in economics, sociology, or international relations and owes much of its modern form to J. von Neumann [1]. The generic situation in game theory consists of a set of players $\{X, Y, \ldots\}$ choosing between different strategies $\left\{X_{i}\right\},\left\{Y_{i}\right\}, \ldots$, the combination of which determines the outcome of a game specified by the payoffs $P_{X}\left(X_{i}, Y_{i}, \ldots\right), P_{Y}\left(X_{i}, Y_{i}, \ldots\right), \ldots$ each player is going to receive. The payoffs depend on the strategies of all players and the problem for every individual player is to choose his strategy such as to optimize his payoff without having control over the strategies of all other players. Despite the extreme simplification of the real world situation inherent in this framework, game theory has proven not only to be a viable mathematical discipline but also to be able to characterize important features of economical systems. Many interesting results have been obtained since von Neumann's pioneering work including the characterization of equilibria [1,2] and the emergence of cooperation [3]. However, detailed investigations have been restricted either to general statements concerning e.g., the existence of equilibria, or to situations where every player has only a small number of strategies at his disposal and where the payoffs are simple functions of these strategies. As many situations of interest show a large number of possible strategies and rather complicated relationships between strategic choices and the resulting payoffs, it is tempting to model the payoffs by a random function and to apply the methods of statistical mechanics to describe the properties of the game. This will be a sensible approach if there are characteristic "macroscopic" quantities which do not depend on the particular realization of the random parameters, i.e. are self-averaging in the sense of the statistical mechanics of disordered systems 酒, for related applications see [5].

In the present letter we show how methods from statistical mechanics can be applied to characterize the statistical properties of optimal strategies in matrix games with large randomly chosen payoff matrices. Explicitly we calculate the mean payoff and the fraction of pure strategies which occur in the optimal mixed strategy of a player. For simplicity we restrict ourselves to matrix games, the type of zero-sum games between two players which also forms the basis of von Neumann's treatment [1],6]. Such games are defined by a (not necessarily square) payoff-matrix $c_{i j}$ : Player $X$ may choose between $N$ strategies $X_{i}$ and player $Y$ between $M$ strategies $Y_{j}$ where $i=1, \ldots, N$ and $j=1, \ldots, M$. At each step of this game they receive the payoffs $P_{X}\left(X_{i}, Y_{j}\right)=$ $-P_{Y}\left(X_{i}, Y_{j}\right)=: c_{i j}$. As player $X$ wishes to gain as large a payoff $c_{i j}$ as possible, whereas player $Y$ must attempt to reach as small a value of $c_{i j}$ in order to maximize his payoff $P_{Y}\left(X_{i}, Y_{j}\right)=-c_{i j}$, the goals of the players are completely conflicting. Thus it is appropriate for the players to proceed as follows: Player $X$ knows that when playing strategy $X_{i}$ he will receive at least the payoff $\min _{j} c_{i j}$. He therefore chooses strategy $X_{i^{*}}$ satisfying $\min _{j} c_{i^{*} j}=\max _{i} \min _{j} c_{i j}$. Equivalently, player $Y$ plays strategy $Y_{j^{*}}$ determined by $\max _{i} c_{i j^{*}}=\min _{j} \max _{i} c_{i j}$ since it minimizes his losses for the optimal choices of $X$. It is easy to show that $\max _{i} \min _{j} c_{i j} \leq \min _{j} \max _{i} c_{i j}$ always. The situation is simple if the matrix has a socalled saddle-point, i.e. if there is a pair $i^{*}, j^{*}$ satisfying $\max _{i} \min _{j} c_{i j}=c_{i^{*} j^{*}}=\min _{j} \max _{i} c_{i j}$. In this case it is optimal for both players to stick to their pure strategies $X_{i^{*}}$ and $Y_{i^{*}}$ respectively, since deviations from an optimal strategy by one of the players will lead to a lower payoff for this player. For a large random matrix $c$ the probability for the existence of a such a saddle point vanishes exponentially with the size of the matrix and the choice of an optimal strategy is less obvious. Since in this case $\max _{i} \min _{j} c_{i j}<\min _{j} \max _{i} c_{i j}$, player $X$ will attempt to achieve a greater gain than his guaranteed minimal gain $\max _{i} \min _{j} c_{i j}$ and likewise $Y$ will attempt to achieve a smaller loss than $\min _{j} \max _{i} c_{i j}$. To this end they have to prevent their opponent from guessing which strategy they are going to play and choose each strategy with a certain probability $x_{i}$ and $y_{j}$ respectively [1]. A vector $x_{i}$ of probabilities is called a mixed strategy and by the normalization condition is constrained to lie on the $\mathrm{N}$ dimensional simplex. The famous minimax theorem by 
von Neumann states that for any payoff matrix $c$ there exists a saddle point of mixed strategies, i.e. there are two vectors $x_{i}^{*}$ and $y_{j}^{*}$ such that

$\max _{\left\{x_{i}\right\}} \min _{\left\{y_{j}\right\}} \sum_{i j} x_{i} c_{i j} y_{j}=\sum_{i j} x_{i}^{*} c_{i j} y_{j}^{*}=\min _{\left\{y_{j}\right\}} \max _{\left\{x_{i}\right\}} \sum_{i j} x_{i} c_{i j} y_{j}$.

The expected payoff for the optimal mixed strategies $\nu_{c}:=\sum_{i j} x_{i}^{*} c_{i j} y_{j}^{*}$ is called the value of the game and $x_{i}^{*}, y_{i}^{*}$ denote optimal mixed strategies of player $X$ and $Y$ since again deviations from an optimal strategy by one of the players will lead to a lower payoff for this player.

In the following we show how the statistical properties of such optimal mixed strategies for random payoff matrices may be characterized analytically in the limit $N \rightarrow \infty, M \rightarrow \infty$ with $M / N=\alpha=O(1)$. As is generally the case in fully connected disordered systems, only the first two cumulants of the probability distribution $P\left(\left\{c_{i j}\right\}\right)$ are relevant. Since an average value $\langle\langle c\rangle\rangle$ of the elements of the payoff matrix only results in a modified value of the game $\nu_{c}+\langle\langle c\rangle\rangle$ without changing the optimal mixed strategies, we may set $\langle\langle c\rangle\rangle=0$ without loss of generality and take the elements $c_{i j}$ to be independent Gaussian distributed variables with zero mean and variance $N^{-1}$.

We then note [6] that a necessary and sufficient condition for the mixed strategy $\left\{x_{i}\right\}$ of player $X$ to be optimal is

$$
\sum_{i} x_{i} c_{i j} \geq \nu_{c} \forall j
$$

The condition is necessary since if violated for some $j$ player $Y$ playing $Y_{j}$ will lead to a payoff lower than $\nu_{c}$. It is also sufficient since combining (1) with the minimax theorem gives $\sum_{i j} x_{i} c_{i j} y_{j}^{*}=\nu_{c}$. We may thus characterize mixed strategies of player $X$ by introducing the partition function

$Z(\nu)=\frac{\prod_{i=1}^{N}\left(\int_{0}^{\infty} d x_{i}\right) \delta\left(\sum_{i=1}^{N} x_{i}-N\right) \prod_{j=1}^{\alpha N} \Theta\left(\sum_{i} x_{i} c_{i j}-\nu\right)}{\prod_{i=1}^{N}\left(\int_{0}^{\infty} d x_{i}\right) \delta\left(\sum_{i=1}^{N} x_{i}-N\right)}$

where $\Theta(x)$ is the Heaviside step-function and the probabilities of playing a given strategy and the payoff have been rescaled so that $\sum_{i=1}^{N} x_{i}=N$ for convenience. Thus $Z(\nu)$ equals the fraction of the simplex obeying $\sum_{i} x_{i} c_{i j} \geq \nu \forall j$ and therefore lies on the interval [0,1]. Since $Z(\nu)$ scales exponentially with $\mathrm{N}$, the quantity central to our calculation is the entropy $S(\nu):=1 / N \ln Z(\nu)$, which in general will be negative as usual for classical systems with continuous degrees of freedom.

Assuming the entropy $S(\nu)$ to be self-averaging, we use the replica-trick $\ln Z=\lim _{n \rightarrow 0} \frac{d}{d n} Z^{n}$ and compute the average over the payoffs of the replicated partition function for integer $n(a, b=1 \ldots n)$. The calculation proceeds by using the integral representation of the Heaviside step-function and by introducing the symmetric matrix of overlap order parameters $q_{a b}=1 / N \sum_{i} x_{i}^{a} x_{i}^{b}$ via integrals over $q_{a b}$ and delta-functions represented by integrals over the conjugate order parameters $\hat{q}_{a b}$ [7]. The integrals over $E_{a}$ arise from the integral representation of the constraint $\sum_{i} x_{i}^{a}=N$ giving

$$
\begin{aligned}
& \left\langle\left\langle Z^{n}(\nu)\right\rangle\right\rangle=\prod_{a \geq b} \int \frac{d q_{a b} d \hat{q}_{a b}}{2 \pi / N} \prod_{a} \int \frac{d E_{a}}{2 \pi / N} \\
& \exp \left(-i N \sum_{a \geq b} q_{a b} \hat{q}_{a b}-i N \sum_{a} E_{a}\right) \\
& \prod_{a, i} \int_{0}^{\infty} d x_{i}^{a} \exp \left(i \sum_{a \geq b, i} \hat{q}_{a b} x_{i}^{a} x_{i}^{b}+i \sum_{a, i} E_{a} x_{i}^{a}\right) \\
& \prod_{a, j} \int_{\nu}^{\infty} d \lambda_{j}^{a} \int \frac{d y_{j}^{a}}{2 \pi} \exp \left(-\frac{1}{2} \sum_{a, b, j} q_{a b} y_{j}^{a} y_{j}^{b}+i \sum_{a, j} y_{j}^{a} \lambda_{j}^{a}\right)
\end{aligned}
$$

In the limit of large payoff matrices $N \rightarrow \infty$ the integrals over order parameters are dominated by their saddle point. Throughout this paper we use the replicasymmetric ansatz \&

$$
\begin{array}{llll}
q_{a a}=q_{1} & i \hat{q}_{a a}=-1 / 2 \hat{q}_{1} & & i E_{a}=E \\
q_{a b}=q_{0} & i \hat{q}_{a b}=\hat{q}_{0} & & \forall a>b .
\end{array}
$$

The limit $n \rightarrow 0$ of (3) may now be taken by analytic continuation giving an entropy

$$
\begin{aligned}
& S(\nu)=\operatorname{extr}_{q_{1}, q_{0}, E, \hat{q}_{1}, \hat{q}_{0}}\left[\frac{1}{2} q_{1} \hat{q}_{1}+\frac{1}{2} q_{0} \hat{q}_{0}-E+\frac{1}{2} \ln (2 \pi)\right. \\
& +\alpha \int D s \ln H\left(\frac{\sqrt{q_{0}} s+\nu}{\sqrt{q_{1}-q_{0}}}\right)-\frac{1}{2} \ln \left(\hat{q}_{1}+\hat{q}_{0}\right)-1 \\
& \left.+\frac{\hat{q}_{0}+E^{2}}{2\left(\hat{q}_{1}+\hat{q}_{0}\right)}+\int D r \ln H\left(-\frac{\sqrt{\hat{q}_{0}} r+E}{\sqrt{\hat{q}_{1}+\hat{q}_{0}}}\right)\right],
\end{aligned}
$$

where $D s=\frac{d s}{\sqrt{2 \pi}} \exp \left(-s^{2} / 2\right)$, and $H(x)=\int_{x}^{\infty} D s$. Numerical evaluation of (5) shows that $S(\nu)$ is a continuously decreasing function of $\nu$. At $\nu_{c}$ it tends to $-\infty$, indicating that for larger values of $\nu$ there are no more solutions to (11). Furthermore as $\nu \rightarrow \nu_{c}$ one finds $q_{0} \rightarrow q_{1}$ indicating that as the points contributing to (2) crowd into an ever decreasing area of the simplex which shrinks to a point at $\nu_{c}$ their mutual overlap $q_{0}$ approaches the self-overlap $q_{1}$.

In this regime the entropy may be conveniently written in terms of the order parameters $q_{0}, \hat{q}_{0}, E, \hat{w}=\hat{q}_{1}+\hat{q}_{0}$ and $v=q_{1}-q_{0}$. For $\nu<\nu_{c} S(\nu)$ describes sub-optimal strategies. As $v \rightarrow 0$ we find $\hat{q}_{0} \sim v^{-2}, \hat{w} \sim v^{-1}$. Rescaling the conjugate order parameters accordingly and expanding the saddle-point equations to leading order in $v$ as $v \rightarrow 0$ we find

$$
\begin{aligned}
\hat{w}-\alpha H\left(-\nu_{c} / \sqrt{q_{0}}\right) & =0 \\
\hat{w}-H\left(-E / \sqrt{\hat{q}_{0}}\right) & =0 \\
\hat{q}_{0}-\left(\nu_{c}^{2}+q_{0}\right) \hat{w}-\alpha \sqrt{q_{0}} \nu_{c} G\left(-\nu_{c} / \sqrt{q_{0}}\right) & =0 \\
q_{0}-\left(E^{2}+\hat{q}_{0}\right) / \hat{w}-\sqrt{q_{0}} E / \hat{w}^{2} G\left(-E / \sqrt{\hat{q}_{0}}\right) & =0
\end{aligned}
$$


with $E=q_{0} \hat{w}-\hat{q}_{0}$.

The statistical properties of optimal strategies $\left\{x_{i}^{\text {opt }}\right\}$ may be deduced from the proportion of strategies $X_{i}$ with $x_{i}>a$

$$
\theta(a):=\left\langle\left\langle 1 / N \sum_{i} \Theta\left(x_{i}^{\mathrm{opt}}-a\right)\right\rangle\right\rangle=H\left(\frac{\hat{w} a-E}{\sqrt{\hat{q}_{0}}}\right) .
$$

Thus only a fraction $\theta(0)=\hat{w}$ of the pure strategies $X_{i}$ have $x_{i}>0$ and are played with non-zero probability. This striking effect may be explained by considering the behaviour of player $Y$, whose optimal mixed strategy $y_{j}^{*}$ obeys $\lambda_{i}^{*}=\sum_{j} c_{i j} y_{j}^{*} \leq \nu_{c} \forall i$. Since $\nu_{c}=1 / N \sum_{i} x_{i}^{*} \lambda_{i}^{*}$, $x_{i}^{*}$ must be zero if $\lambda_{i}^{*}<\nu_{c}$. This mechanism thus ensures an expected payoff $\nu_{c}$ to $X$, even if $Y$ chooses an optimal strategy. However it is not to be confused with the concept of domination, widely discussed in the game theory literature [1,66,9], where a strategy $X_{i}$ has $x_{i}=0$ because whatever the response of the opponent some other pure or mixed strategy will lead to a higher expected payoff. In fact in the thermodynamic limit domination of a pure strategy occurs with probability zero since for a pure strategy $X_{k}$ to be dominated by a mixed strategy $x_{i}^{D}$ requires $1 / N \sum_{i} x_{i}^{D} c_{i j} \geq c_{k j} \forall j$ but the lhs is $O\left(N^{-1}\right)$ whereas the rhs is $O\left(N^{-1 / 2}\right)$.

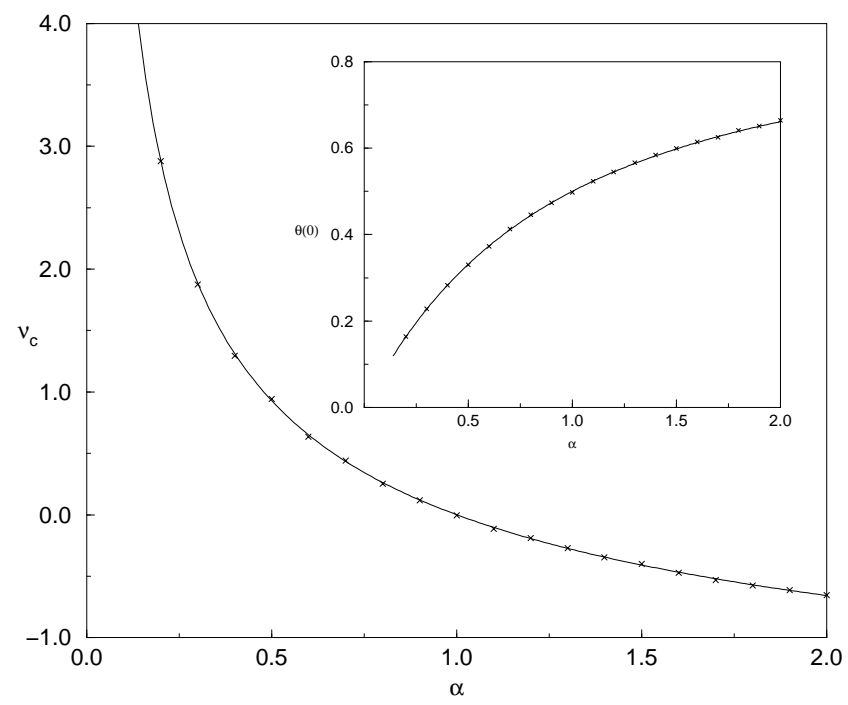

FIG. 1. The value of the game $\nu_{c}$ and (inset) the fraction of strategies played with non-zero probability $\theta(0)$ as a function of $\alpha$. The analytical results (full line) are compared to numerical simulations with $\mathrm{N}=200$ averaged over 200 samples. The symbol size corresponds to the statistical error.

Figure 1 shows the value of the game and (inset) the fraction of strategies played with non-zero probability as a function of the aspect ratio $\alpha$ of the payoff matrix. At $\alpha=1 \nu_{c}=0$ and $\theta(0)=1 / 2$. The result $\nu_{c}=0$ at $\alpha=1$ is a consequence of the symmetry of the distribution of payoffs under $c_{i j} \rightarrow-c_{j i}$, i.e. under interchange of player $X$ and player $Y[10]$. For $\alpha>1$ player $Y$ has a greater choice of strategies than player $X$ and vice versa. As expected, the payoff to player $X$ decreases as the range of strategy choices of player $Y$ increases. The fraction of strategies played with non-zero probability increases with $\alpha$, which reflects the decrease of $\nu_{c}$ with $\alpha$ : At lower $\nu_{c}$ there are fewer $i$ with $\lambda_{i}^{*}=\sum_{j} c_{i j} y_{j}^{*}<\nu_{c}$, so as argued above the number of strategies $X_{i}$ played with non-zero probability increases as a result.

We next abandon the initial assumption that the individual entries $c_{i j}$ in the payoff-matrix are independently distributed and consider the case where the outcomes of the game for different strategy choices of the players are correlated with each other. Such correlations may arise quite naturally in real applications since we expect some strategies to have broadly similar properties and hence yield similar results for a given response of the respective opponent. For simplicity we restrict the discussion to the case $\alpha=1$. The most general tractable case appears to be

$$
\left\langle\left\langle c_{i j} c_{k l}\right\rangle\right\rangle /\left(\left\langle\left\langle c_{i j}\right\rangle\right\rangle\left\langle\left\langle c_{k l}\right\rangle\right\rangle\right)=: \mathcal{C}_{(i j)(k l)}=\mathcal{C}_{i k}^{c} \mathcal{C}_{j l}^{r}
$$

where $\mathcal{C}_{i k}^{c}$ and $\mathcal{C}_{j l}^{r}$ refer to column- and row-like correlations. Of course $P\left(\left\{c_{i j}\right\}\right)$ is not uniquely determined by its second moments, but as argued above it suffices to consider Gaussian distributed payoff matrices so

$$
P\left(\left\{c_{i j}\right\}\right)=\frac{1}{\sqrt{(2 \pi)^{N^{2}}\|\mathcal{C}\|}} \exp \left(-N / 2 \sum_{i j k l} c_{i j} \mathcal{C}^{-1}{ }_{(i j)(k l)} c_{k l}\right)
$$

The specific form of $\mathcal{C}_{i k}^{c}$ and $\mathcal{C}_{j l}^{r}$ we will consider in the following is

$$
\mathcal{C}_{i k}^{c, r}= \begin{cases}1 & i=k \\ c_{c, r} / N & i \neq k\end{cases}
$$

and the resulting replica symmetric entropy averaged over the distribution (9) may be calculated as outlined for the case of uncorrelated payoffs above. Again in the limit $v \rightarrow 0$ the corresponding saddle point equations describe optimal strategies. For $c_{c}=c_{r}=c$ the optimal payoff is zero as a result of the symmetry of (9) under the exchange of players. Figure 2 shows the fraction $\theta(0)$ of strategies played with non-zero probability in optimal strategies as a function of $c . \theta(0)$ decreases with increasing $c$ : At positive $c$ there are strategies which tend to be beneficial for player $X$ whatever the response of the opponent. As a result $X$ concentrates on a smaller fraction of his strategies and vice versa for negative $c$.

In the asymmetric case $c_{r}=c, c_{c}=0$ however a nonzero value of the game is possible. The resulting value for $\nu_{c}$ and the fraction of strategies played with non-zero probability are shown in figure 3 . Again the correlation between payoffs in the same row of the payoff matrix lead to strategies which tend to be either beneficial or detrimental to player $X$. 


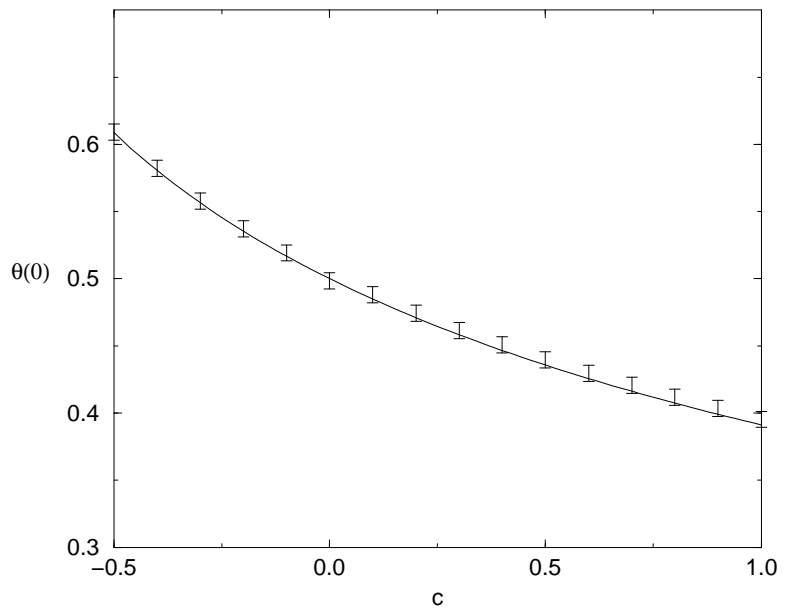

FIG. 2. The fraction $\theta(0)$ strategies played with non-zero probability as a function of $c=c_{r}=c_{c}$.

By admitting only the beneficial ones into his mixed strategies, $X$ may achieve a positive payoff. The fraction of strategies played with non-zero probability decreases accordingly. For negative $c$ the entries in the same rows of the payoff matrix are anticorrelated, so different responses of player $Y$ to the same strategy of player $X$ tend to lead to different payoffs. This situation leads to a negative value of the game.

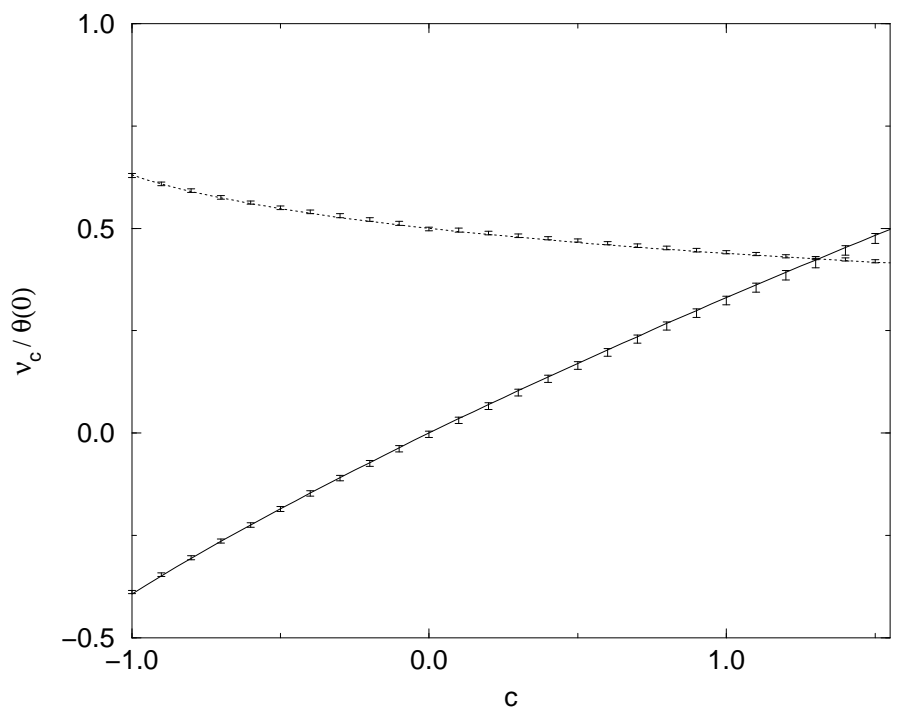

FIG. 3. The optimal payoff $\nu_{c}$ (full) and the fraction $\theta(0)$ of strategies played with non-zero probability (dotted) against $c=c_{r}$ at $c_{c}=0$.

The simulation results shown in figures 1-3 were obtained using the simplex-algorithm to solve the linear programming problem [6] defined by (11) for a system of size $N=200$ averaged over 200 payoff matrices with Gaussian distributed inputs [11. The numerical results show very good agreement with the analytical expressions.

In conclusion we have shown that techniques from the statistical mechanics of disordered systems may be used to analyze the statistical properties of optimal solutions of matrix games with random payoffs. Selfaveraging "macroscopic" quantities such as the value of the game were identified and calculated for various probability distributions. These quantities include the fraction of strategies played with non-zero probability. Further problems in matrix games which may be treated using these methods include the effects of deviating from the optimal strategy and the influence of perturbations of the payoff matrix on the optimal strategy, which forms the basis of the justification for the full stability of mixed equilibria 12]. Furthermore work is in progress on a field of interest to current mathematical game theory, the statistical description of Nash-equilibria in bimatrix games.

Acknowledgments: It is a pleasure to thank $\mathrm{R}$. Monasson, L. Schneider, and M. Weigt for stimulating discussions. JB gratefully acknowledges financial support by the Studienstiftung des Deutschen Volkes.

[1] J. von Neumann, and O. Morgenstern Theory of Games and Economic Behavior (3rd edn) (Princton Press, Princton, 1953)

[2] J.F. Nash, Annals of Mathematics, 54(2), 286 (1951)

[3] R. Axelrod The Evolution of Cooperation (Basic Books, New York, 1984)

[4] M. Mézard, G. Parisi, and M. A. Virasoro Spin Glass Theory and Beyond (World Scientific, Singapore, 1987)

[5] Y. Fu, and P.W. Anderson, J. Phys. A19, 1605 (1986), S. Diederich, and M. Opper, Phys. Rev. A39(8), 4333 (1989), R. Monasson, and R. Zecchina, Phys. Rev. Lett. 76(21),3881 (1996), S. Galluccio, J.-P. Bouchaud, and M. Potters, cond-mat/9801209, A. Crisanti et. al., Products of Random Matrices in Statistical Physics (Springer, Berlin, 1993)

[6] W. Jianhua The Theory of Games (Oxford University Press, 1988)

[7] E. Gardner, J. Phys. A21, 257 (1988)

[8] Replica symmetry may be justified from the convexity of the solution space of equation (1), furthermore it has been found to be locally stable for $\nu<\nu_{c}$.

[9] A. Brandenburger, Journal of Economic Perspectives, 6(4), 83 (1992)

[10] Under $\alpha \rightarrow 1 / \alpha \nu_{c} \rightarrow-\sqrt{\alpha} \nu_{c}$ since the variance of the payoffs is $N^{-1}$.

[11] Virtually identical results have been obtained for different probability ensembles such as $c_{i j}= \pm N^{-1 / 2}$.

[12] J.C. Harsanyi, Int. J. of Game Theory 2(1),1(1973) 